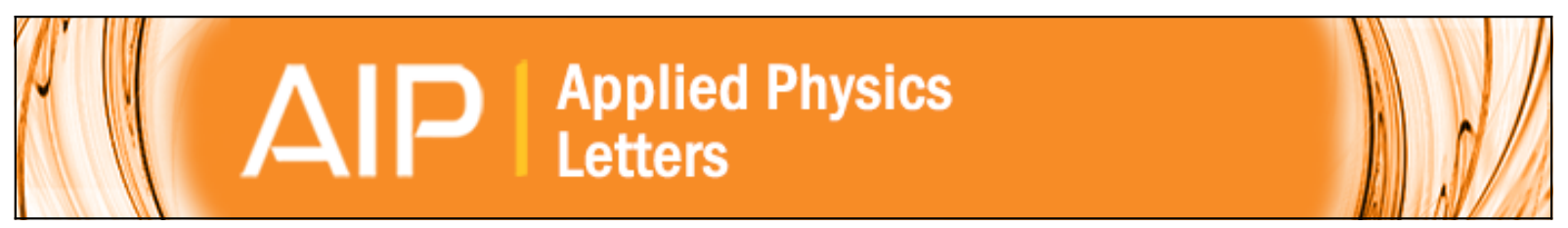

\title{
Investigation of the optical properties of MoS2 thin films using spectroscopic ellipsometry
}

Chanyoung Yim, Maria O'Brien, Niall McEvoy, Sinéad Winters, Inam Mirza, James G. Lunney, and Georg S. Duesberg

Citation: Applied Physics Letters 104, 103114 (2014); doi: 10.1063/1.4868108

View online: http://dx.doi.org/10.1063/1.4868108

View Table of Contents: http://scitation.aip.org/content/aip/journal/apl/104/10?ver=pdfcov

Published by the AIP Publishing

\section{Articles you may be interested in}

Optical properties and phase change transition in Ge 2 Sb 2 Te 5 flash evaporated thin films studied by temperature dependent spectroscopic ellipsometry

J. Appl. Phys. 104, 043523 (2008); 10.1063/1.2970069

Optical properties of amorphous Ga As $1 \times \mathrm{N}$ x film sputtering with different $\mathrm{N} 2$ partial pressures

J. Vac. Sci. Technol. A 24, 1714 (2006); 10.1116/1.2217977

Effect of metal-ion doping on the optical properties of nanocrystalline $\mathrm{ZnO}$ thin films

J. Appl. Phys. 99, 014306 (2006); 10.1063/1.2158503

Anisotropic optical properties and molecular orientation in vacuum-deposited ter(9,9-diarylfluorene)s thin films using spectroscopic ellipsometry

J. Appl. Phys. 95, 881 (2004); 10.1063/1.1635991

Optical characterization of Culn $1 \times$ Ga $x$ Se 2 alloy thin films by spectroscopic ellipsometry J. Appl. Phys. 94, 879 (2003); 10.1063/1.1581345

\section{AIP $\mid$ chaos CALL FOR APPLICANTS Seeking new Editor-in-Chief}




\title{
Investigation of the optical properties of $\mathrm{MoS}_{2}$ thin films using spectroscopic ellipsometry
}

\author{
Chanyoung Yim, ${ }^{1,2}$ Maria O’Brien, ${ }^{1,2}$ Niall McEvoy, ${ }^{2}$ Sinéad Winters, ${ }^{1,2}$ Inam Mirza,,${ }^{2,3}$ \\ James G. Lunney, ${ }^{2,3}$ and Georg S. Duesberg ${ }^{1,2,4, a)}$ \\ ${ }^{1}$ School of Chemistry, Trinity College Dublin, Dublin 2, Ireland \\ ${ }^{2}$ Centre for Research on Adaptive Nanostructures and Nanodevices (CRANN), Trinity College Dublin, \\ Dublin 2, Ireland \\ ${ }^{3}$ School of Physics, Trinity College Dublin, Dublin 2, Ireland \\ ${ }^{4}$ Advanced Materials and BioEngineering Research (AMBER) Centre, Trinity College Dublin, \\ Dublin 2, Ireland
}

(Received 9 January 2014; accepted 26 February 2014; published online 14 March 2014)

\begin{abstract}
Spectroscopic ellipsometry (SE) characterization of layered transition metal dichalcogenide (TMD) thin films grown by vapor phase sulfurization is reported. By developing an optical dispersion model, the extinction coefficient and refractive index, as well as the thickness of molybdenum disulfide $\left(\mathrm{MoS}_{2}\right)$ films, were extracted. In addition, the optical band gap was obtained from SE and showed a clear dependence on the $\mathrm{MoS}_{2}$ film thickness, with thinner films having a larger band gap energy. These results are consistent with theory and observations made on $\mathrm{MoS}_{2}$ flakes prepared by exfoliation, showing the viability of vapor phase derived TMDs for optical applications. (C) 2014 AIP Publishing LLC. [http://dx.doi.org/10.1063/1.4868108]
\end{abstract}

In recent years, there has been a large volume of research conducted on two dimensional (2D) materials such as graphene and transition metal dichalcogenides (TMDs) due to their suitability for future electronic/optoelectronic device applications. ${ }^{1-4}$ In particular, molybdenum disulfide $\left(\mathrm{MoS}_{2}\right)$, a semiconducting layered TMD, has been identified as one of the most promising 2D materials for nano-electronic applications because of its properties which can be tuned by controlling the number of layers or by careful selection of substrate/gate dielectric materials. ${ }^{5-8}$ Moreover, unlike pristine graphene which has no band gap, monolayer $\mathrm{MoS}_{2}$ has a direct band gap of $\sim 1.8 \mathrm{eV}$ and bulk $\mathrm{MoS}_{2}$ has an indirect band gap of $\sim 1.3 \mathrm{eV},{ }^{5}$ and electronic devices based on monoor multilayered $\mathrm{MoS}_{2}$ films have shown good photodetection capability. ${ }^{7,9}$ While mechanical exfoliation is a widely used method to prepare layered $\mathrm{MoS}_{2}$ thin films, the difficulty of controlling layer thickness and the lateral size limitation have led to the development of alternative synthesis routes. Recently, vapor-phase growth methods, whereby sulfur powder is vaporized and reacted with thin molybdenum or molybdenum oxide seed layers, have been introduced. ${ }^{10-12}$ This makes it possible to obtain large-area $\mathrm{MoS}_{2}$ thin films with tunable thickness and such films have been employed to fabricate various types of nano-optoelectronic devices including solar cells, sensors, and phototransistors. ${ }^{13-15}$

For optoelectronic device applications of $\mathrm{MoS}_{2}$ to be fully understood, it is necessary to know the refractive index $(n)$ and the extinction coefficient $(k)$. Spectroscopic ellipsometry (SE) is a powerful non-destructive technique to measure the optical properties of thin films. In SE, the wavelength dependent optical constants and the thickness of thin films can be determined by analyzing the change in the polarization state of the reflected light from the film surface and developing an optical dispersion model of the film

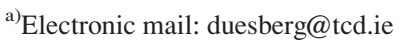

material. ${ }^{16}$ It is frequently used in industry for film characterization and in in-situ thickness monitoring because it is fast, cheap, and non-destructive. In recent years, SE has been employed to investigate the optical constants of graphene, ${ }^{17-20}$ however, to date, SE characterization of 2D layered TMDs, such as $\mathrm{MoS}_{2}$, has rarely been reported.

In this work, we report the measurement of the optical properties of $\mathrm{MoS}_{2}$ thin films with different thicknesses using SE. The $\mathrm{MoS}_{2}$ thin films were synthesized on substrates using a vapor phase sulfurization process and characterized by UV-visible absorption spectroscopy and Raman spectroscopy. Based on SE measurements, an optical dispersion model was developed for the $\mathrm{MoS}_{2}$ thin films, and the optical constants $(n, k)$ and the film thickness values were extracted. In addition, optical band gap values of the $\mathrm{MoS}_{2}$ thin films were obtained from analysis of the $k$ values. The values are consistent with data derived from other techniques and theoretical values. This work establishes SE as a viable tool for the characterization of TMDs. Further, our study shows that vapor phase derived $\mathrm{MoS}_{2}$ exhibits similar optical properties to exfoliated materials, making it a strong candidate for the manufacture for optoelectronic devices.

Vapor phase sulfurization was employed to synthesize $\mathrm{MoS}_{2}$ thin films, as described in the previous work. ${ }^{11,14} \mathrm{Mo}$ (99.99\%, MaTecK) films with various thicknesses (1-20 nm) were deposited by sputtering on fused quartz substrates $(\sim 10 \mathrm{~mm} \times 10 \mathrm{~mm})$ and on commercially available silicon dioxide $\left(\mathrm{SiO}_{2}, \sim 290 \mathrm{~nm}\right.$ thick) substrates $(\sim 10 \mathrm{~mm} \times 10 \mathrm{~mm})$, which were thermally grown on top of $\langle 100\rangle$ oriented crystalline silicon ( $\mathrm{Si}$ ) wafers, using a Gatan Precision Etching and Coating System (PECS). A quartz crystal microbalance was used to monitor the Mo film deposition rate $(<0.1 \mathrm{~nm} / \mathrm{s})$ and thickness. The Mo samples were sulfurized in a quartz tube furnace consisting of two different heating zones as shown in Figure 1(a). The samples were loaded into the hot zone which was heated to $750^{\circ} \mathrm{C}$ and annealed for $30 \mathrm{~min}$ at a pressure of 
(a)

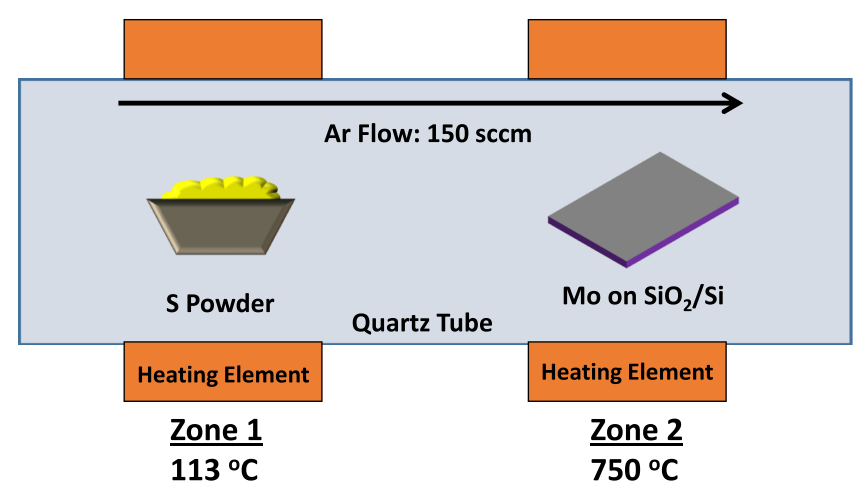

(b)

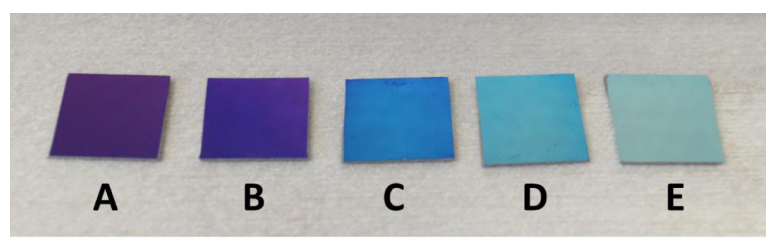

FIG. 1. (a) Schematic diagram of the vapor phase sulfurization process used for $\mathrm{MoS}_{2}$ thin film synthesis. (b) Photograph of $\mathrm{MoS}_{2}$ samples with increasing thickness (A-E).

$\sim 1$ Torr with an argon (Ar) flow of $150 \mathrm{sccm}$ (standard cubic centimeters per minute). Sulfur vapor was then produced by heating sulfur powder (MaTecK, 99\%) up to its melting point $\left(\sim 113^{\circ} \mathrm{C}\right)$ in the second upstream heating zone of the furnace and transported to the Mo samples using $\mathrm{Ar}$ gas as carrier. After sulfurization, the samples were annealed for $30 \mathrm{~min}$ at $750^{\circ} \mathrm{C}$ and then cooled down to room temperature. Raman spectroscopy measurements were carried out with a HORIBA Jobin-Yvon Labram HR, using an excitation wavelength of $532 \mathrm{~nm}$ and an 1800 lines/mm grating. UV-visible absorption data were obtained with a Varian Cary 6000i double beam spectrophotometer in the spectral range of $200-800 \mathrm{~nm}$. The SE characterization of the $\mathrm{MoS}_{2}$ thin films was conducted using an Alpha SE tool (J. A. Woollam Co., Inc.) operating in the wavelength range of $380-900 \mathrm{~nm}(1.38-3.26 \mathrm{eV})$ at an angle of incidence of $70^{\circ}$ with a beam spot size of $\sim 40 \mathrm{~mm}^{2}$ and a rotating compensator. SE data were analyzed using CompleteEASE 4.72 (J. A. Woollam Co., Inc.). X-ray reflectivity (XRR) measurements were conducted using a Bruker D8-Discover X-ray diffractometer which is fitted with $\mathrm{Cu}$ $\mathrm{K}$-alpha X-ray source and Goebel mirror to produce a parallel beam. Thickness values were obtained by fitting the XRR data using a computer simulation called LEPTOS.

In our synthesis, vaporized sulfur reacts with predeposited Mo layers, yielding continuous $\mathrm{MoS}_{2}$ thin films. An optical image of $\mathrm{MoS}_{2}$ thin films on $\mathrm{SiO}_{2} / \mathrm{Si}$ substrates (samples A, B, C, D, and E) is shown in Figure 1(b). The samples are clearly distinguishable from each other due to the surface color difference caused by their varying thicknesses. The films appear homogeneous over the sample area of $\sim 10 \mathrm{~mm} \times 10 \mathrm{~mm}$.

The optical properties of the $\mathrm{MoS}_{2}$ thin films were measured by SE. The raw SE data comprise a tabulation versus wavelength of the amplitude ratio ( $\Psi$, psi) and the phase difference ( $\Delta$, delta) between the $\mathrm{p}$ - and s-polarized components of the reflected light. The two parameters measured by a SE system are related to the ratio $\rho$, defined by the equation of $\rho=r_{p} / r_{s}=\tan (\Psi) \exp (\mathrm{i} \Delta)$, where $r_{p}$ and $r_{s}$ are the amplitude reflection coefficients for the p-polarized and s-polarized light, respectively. ${ }^{16} \mathrm{~A}$ four-layer optical model which consists of a $\mathrm{Si}$ substrate, an interface layer between $\mathrm{Si}$ and $\mathrm{SiO}_{2}, \mathrm{SiO}_{2}$ layer, and a $\mathrm{MoS}_{2}$ layer was built to analyze the $\mathrm{SE}$ spectra. Each layer of the model has several fitting variables such as thickness and optical dispersion model parameters. A value of the goodness of fit, which quantifies how well the data generated by the optical model $(G)$ fit the experimental data $(E)$, is derived from the root mean squared error (MSE) as defined by

$$
M S E=\sqrt{\frac{1}{3 m-l} \sum_{i=1}^{m}\left[\left(N_{E_{i}}-N_{G_{i}}\right)^{2}+\left(C_{E_{i}}-C_{G_{i}}\right)^{2}+\left(S_{E_{i}}-S_{G_{i}}\right)^{2}\right]} \times 1000,
$$

where $m$ is the number of wavelengths measured, $l$ is the number of fit parameters, and $N=\cos (2 \Psi), C=\sin (2 \Psi) \cos (\Delta)$, $S=\sin (2 \Psi) \sin (\Delta){ }^{21}$ The Levenberg-Marquardt nonlinear regression algorithm is used to minimize the MSE during the fitting process.

In a multilayer structure model, it is important to define the optical response of underlying layers in order to minimize optical correlation effects between layers and find more accurate values for the optical constants and the thickness of the top layer. Therefore, SE spectra of reference $\mathrm{SiO}_{2} / \mathrm{Si}$ substrates, where Mo layers were not deposited, were first measured and analyzed using a three-layer optical model composed of a Si substrate, an interface layer between $\mathrm{Si}$ and $\mathrm{SiO}_{2}$, and a $\mathrm{SiO}_{2}$ layer. It is assumed that the underlying layers are stable with well-established optical constants. ${ }^{22}$ The crystalline Si substrate of the model can be presumed to have a semi-infinite thickness, so the thicknesses of the interface and $\mathrm{SiO}_{2}$ layers were determined from the fitting process. Then, the $\mathrm{MoS}_{2}$ layer is added on top of the $\mathrm{SiO}_{2}$ layer in the model structure, allowing its optical properties to be characterized in the presence of well-defined underlying layers. A Tauc-Lorentz (T-L) oscillation model was used to determine the optical properties of the $\mathrm{MoS}_{2}$ thin film. The complex dielectric function of the energy $(E)$ 
(a)

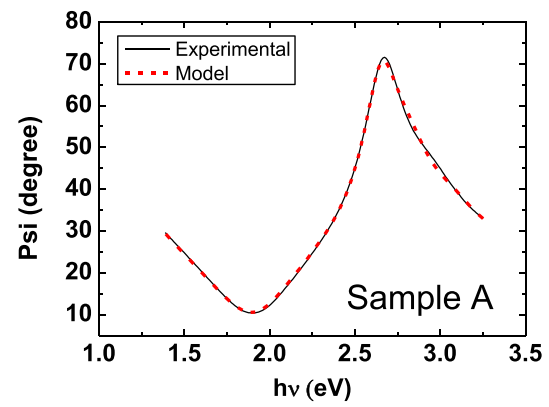

(c)

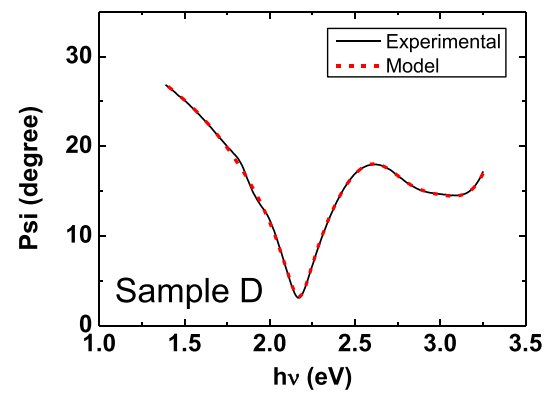

(b)

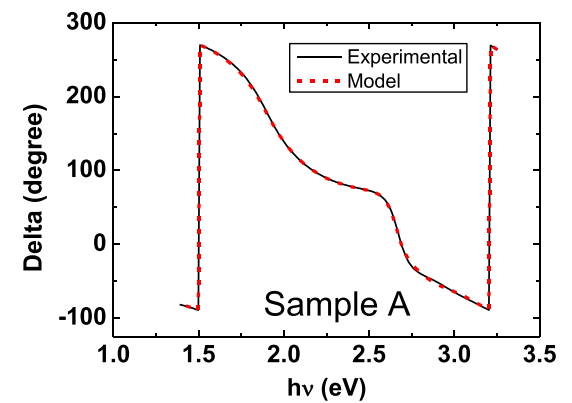

(d)

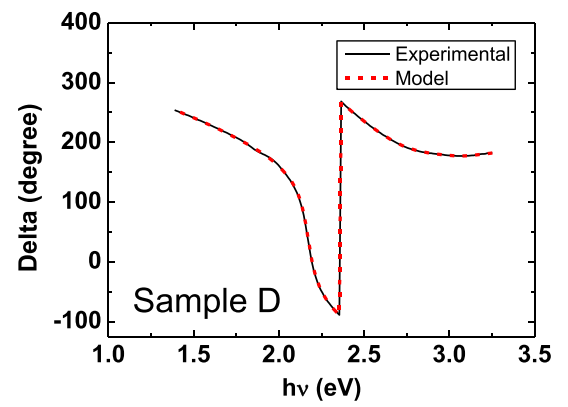

FIG. 2. Plots of the experimental and simulated (model) SE spectra (psi and delta) of $\mathrm{MoS}_{2}$ samples A and D: (a) $\Psi$ and (b) $\Delta$ data of sample A, and (c) $\Psi$ and (d) $\Delta$ data of sample D. is defined as $\varepsilon(E)=\varepsilon_{1}+i \varepsilon_{2}$. In the T-L model, $\varepsilon_{1}$ and $\varepsilon_{2}$ are defined as

$$
\begin{gathered}
\varepsilon_{2}=\left[\frac{A E_{0} C\left(E-E_{b}\right)^{2}}{\left(E^{2}-E_{0}^{2}\right)^{2}+C^{2} E^{2}} \times \frac{1}{E}\right] \text { for } E>E_{b}, \\
\varepsilon_{2}=0 \text { for } E \leq E_{b}, \\
\varepsilon_{1}=\varepsilon_{1}(\infty)+\frac{2}{\pi} P \int_{E_{b}}^{\infty} \frac{\xi \varepsilon_{2}(\xi)}{\xi^{2}-E^{2}} d \xi,
\end{gathered}
$$

where $A, E_{0}, C$, and $E_{b}$ represent the amplitude, center energy, broadening, and band gap of the oscillator, respectively, which all have units of energy $(\mathrm{eV})$, and $P$ stands for the Cauchy principal part of the integral. ${ }^{23}$ The thickness of $\mathrm{MoS}_{2}$ and the fitting parameters of the T-L oscillation model are varied, until the best fit between experimental spectra and simulated data from the optical model is achieved with the lowest MSE values. The experimental and simulated spectra ( $\Psi$ and $\Delta$ ) for $\mathrm{MoS}_{2}$ samples A and D are depicted in Figure 2, showing a good match between them. Additional spectral data for other samples (B, C, and E) are presented in Figure S1 of the supplementary material. ${ }^{28}$ Extracted $\mathrm{MoS}_{2}$ thickness values of the five samples (A, B, C, D, and E) are $1.99 \pm 0.01 \mathrm{~nm}$, $3.01 \pm 0.07 \mathrm{~nm}, 5.53 \pm 0.08 \mathrm{~nm}, 9.83 \pm 0.04 \mathrm{~nm}$, and 19.88 $\pm 0.05 \mathrm{~nm}$, respectively. More details on fitting parameter values of the T-L oscillation model for the best fit are listed in the supplementary material (Table $\mathrm{S} 1) .{ }^{28}$ In addition, XRR measurements of the samples were conducted to verify the $\mathrm{MoS}_{2}$ film thickness, and the measured values were consistent with those extracted from SE measurements. The thickness values of the $\mathrm{MoS}_{2}$ thin films from SE and XRR measurements are summarized in Table I. More details on XRR measurements are presented in the supplementary material. ${ }^{28}$ Figures 3(a) and 3(b) show $n$ and $k$ values of the $\mathrm{MoS}_{2}$ layer for each sample derived from the optical model with the best fits. Two dominant peaks are observed at the energy of $\sim 2.0 \mathrm{eV}$ and $\sim 2.9 \mathrm{eV}$ in the $k$ plot. In addition, an absorbance spectrum of a $\mathrm{MoS}_{2}$ thin film $(\sim 20 \mathrm{~nm})$ on a quartz substrate was measured, as shown in the inset of Figure 3(b). Two spectral regions with dominant absorption variations are observed in the photon energy range of $1.5 \mathrm{eV}-3.8 \mathrm{eV}$, which are related to $\mathrm{A} / \mathrm{B}$ excitonic peaks in the range of $1.8-2.0 \mathrm{eV}$, and $\mathrm{C} / \mathrm{D}$ excitonic peaks in the range of $2.7-3.1 \mathrm{eV}$, respectively. ${ }^{24}$ This shows good agreement with the two $k$ peaks observed at energies of $\sim 2.0 \mathrm{eV}$ and $\sim 2.9 \mathrm{eV}$ in the $k$ plot.

Raman spectra were measured from the $\mathrm{MoS}_{2}$ thin films on the $\mathrm{SiO}_{2} / \mathrm{Si}$ substrates. When probed with a $532 \mathrm{~nm}$ excitation laser, bulk $\mathrm{MoS}_{2}$ has two well-known Raman bands at $\sim 383 \mathrm{~cm}^{-1}$ and $\sim 408 \mathrm{~cm}^{-1}$ which correspond to $E_{2 g}^{1}$ and $A_{1 g}$ vibrational modes, respectively. ${ }^{25}$ Raman spectra of five films, with different thicknesses, are shown in Figure 4. It is evident that the $E_{2 g}^{1}$ and $A_{1 g}$ peaks shift closer to one another with decreasing film thickness. This is consistent with the

TABLE I. Summary of the $\mathrm{MoS}_{2}$ thickness values from SE data and XRR measurements for the five $\mathrm{MoS}_{2}$ samples (samples A, B, C, D, and E). Thickness values of samples A and B from XRR measurements are not available due to the thickness resolution limit of XRR.

\begin{tabular}{lcccccc}
\hline \hline & Sample & A & B & C & D \\
\hline SE & Thickness (nm) & $1.99 \pm 0.01$ & $3.01 \pm 0.07$ & $5.53 \pm 0.08$ & $9.83 \pm 0.04$ & $19.88 \pm 0.05$ \\
& MSE & 4.100 & 7.067 & 3.606 & 5.429 & 5.173 \\
XRR & Thickness (nm) & N.A. & N.A. & $4.4 \pm 1.4$ & $9.9 \pm 1.6$ & $19.4 \pm 1.6$ \\
\hline \hline
\end{tabular}



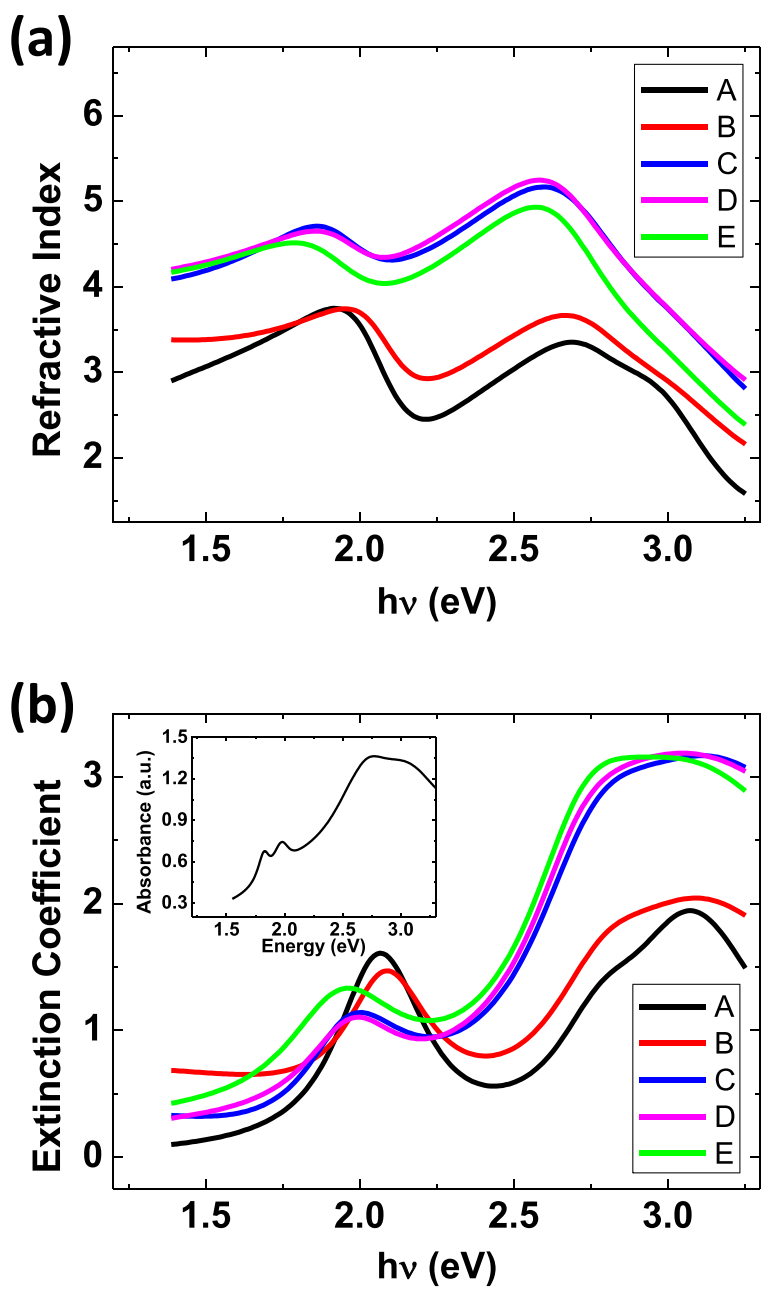

FIG. 3. Plots of (a) the refractive index $n$ and (b) extinction coefficient $k$ values of the $\mathrm{MoS}_{2}$ layers for the five samples (A, B, C, D and E) derived from the optical model with the best fits. The inset of (b) shows a UV-visible absorption spectrum of a $\mathrm{MoS}_{2}$ thin film on a quartz substrate.

report of Li et al., ${ }^{25}$ who described the layer thickness dependence of the Raman signal of mechanically exfoliated $\mathrm{MoS}_{2}$ with decreasing layer thicknesses. In the case of sample E, the $E_{2 g}^{1}$ and $A_{1 g}$ peaks are seen at $384.2 \mathrm{~cm}^{-1}$ and $409.6 \mathrm{~cm}^{-1}$, a separation of $25.4 \mathrm{~cm}^{-1}$, whereas for sample A, the same peaks manifest at $387.0 \mathrm{~cm}^{-1}$ and $407.1 \mathrm{~cm}^{-1}$, a separation of $20.1 \mathrm{~cm}^{-1}$. This suggests that sample A

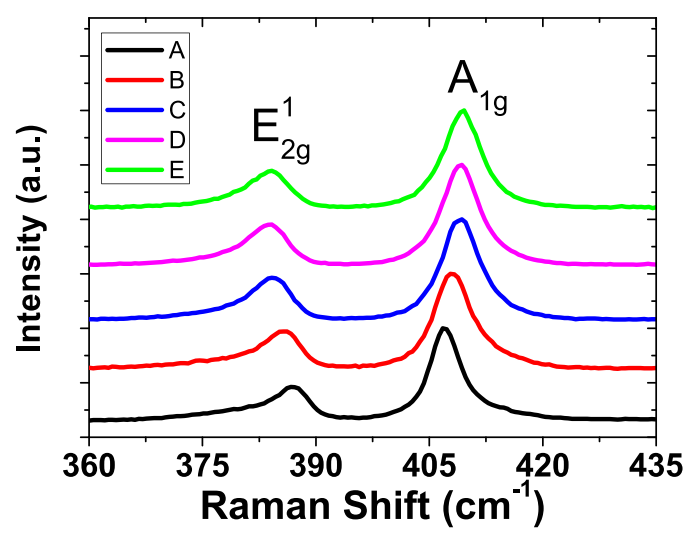

FIG. 4. Raman spectra of the $\mathrm{MoS}_{2}$ thin films on the $\mathrm{SiO}_{2} / \mathrm{Si}$ substrates (samples A, B, C, D, and E). consists of very few (1-3) layers, whereas sample E behaves like bulk $\mathrm{MoS}_{2}$.

Furthermore, studies of the optical band gap energy of the $\mathrm{MoS}_{2}$ thin films were conducted. The optical absorption coefficient $(\alpha)$ of $\mathrm{MoS}_{2}$ thin films can be calculated from the extinction coefficient $k$ and wavelength $\lambda$ by means of $\alpha=4 \pi k / \lambda$. The relationship between absorption coefficient $\alpha$ and the photon energy can be expressed by

$$
\alpha=\frac{K\left(h \nu-E_{g}\right)^{m}}{h \nu},
$$
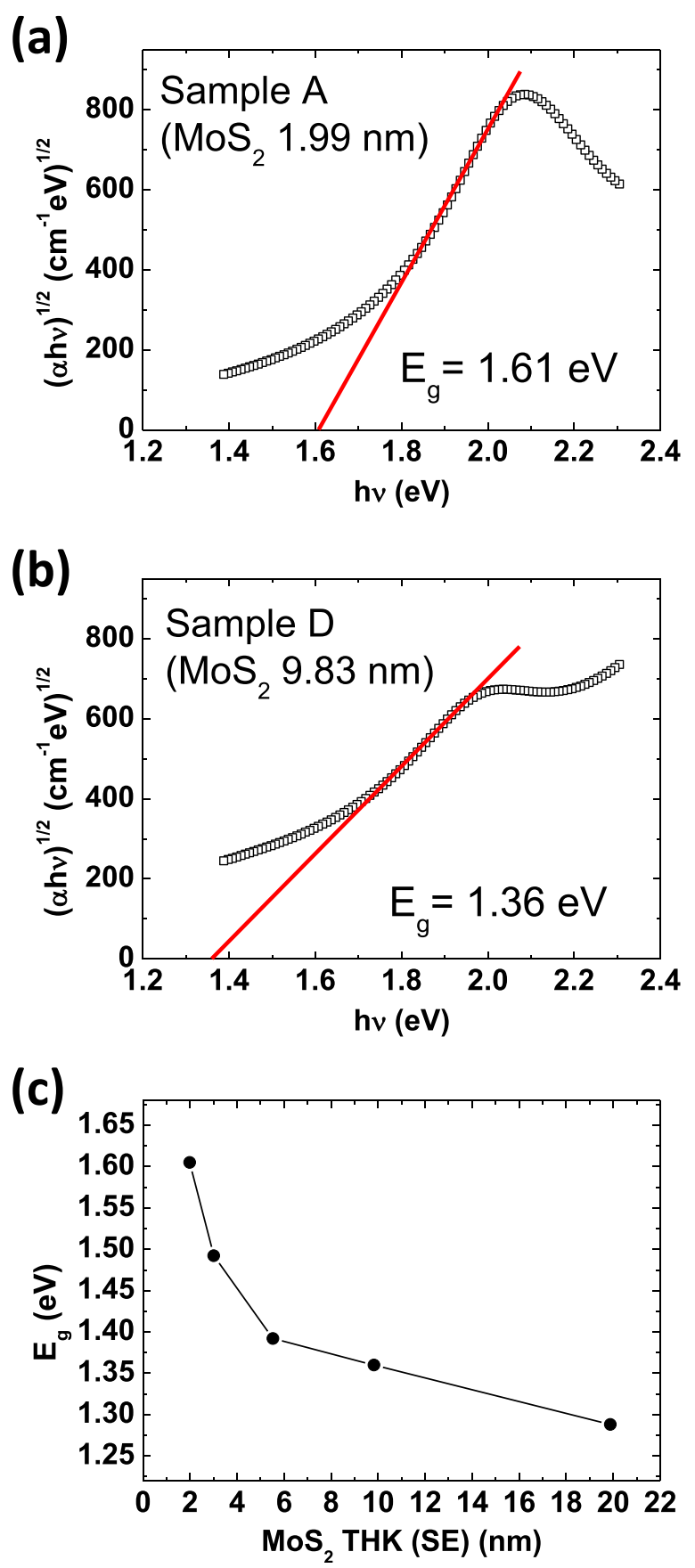

FIG. 5. (a) Plot of $(\alpha h \nu)^{1 / 2}$ vs. $h \nu$ for sample A. (b) Plot of $(\alpha h \nu)^{1 / 2}$ vs. $h \nu$ for sample D. The extrapolation of the linear region to $(\alpha h \nu)^{1 / 2}=0$ (red line) gives the $E_{g}$ value. (c) Plot of $E_{g}$ vs. $\mathrm{MoS}_{2}$ thickness for samples A, B, C, D, and $\mathrm{E}$. 
where $K$ is a constant, $h \nu$ is the incident photon energy, and $m$ is a number that characterizes the transition process. ${ }^{26,27}$ Depending on the transition type, $m$ has a value of $1 / 2$ for direct transition and 2 for indirect transition. Since our $\mathrm{MoS}_{2}$ thin films are considered as multilayer $\mathrm{MoS}_{2}$ films with thicknesses of $1.99-19.88 \mathrm{~nm}$, it is believed that they have primarily indirect band gap transitions. ${ }^{5}$ Thus, the variation of $\alpha$ with $h \nu$ can be determined by using the relation of $(\alpha h \nu)^{1 / 2} \sim\left(h \nu-E_{g}\right)$ and the optical band gap $E_{g}$ can be extracted by extrapolating the linear region of a $(\alpha h \nu)^{1 / 2}$ vs. $h \nu$ plot to $(\alpha h \nu)^{1 / 2}=0$. Plots of $(\alpha h \nu)^{1 / 2}$ vs. $h \nu$ for the $\mathrm{MoS}_{2}$ thin films (samples A and D) are shown in Figures 5(a) and 5(b). The extracted $E_{g}$ values of the five samples are 1.61, $1.49,1.45,1.36$, and $1.29 \mathrm{eV}$ for samples A, B, C, D, and E, respectively. Additional plots of $(\alpha h \nu)^{1 / 2}$ vs. $h \nu$ for samples B, C, and E are shown in Figure S3 of the supplementary material. ${ }^{28}$ It is evident that the thinner $\mathrm{MoS}_{2}$ films have a larger $E_{g}$, as presented in Figure 5(c). The thinnest $\mathrm{MoS}_{2}$ film (sample A) with a measured thickness of $1.99 \mathrm{~nm}$ shows the largest $E_{g}(1.61 \mathrm{eV})$, and as the $\mathrm{MoS}_{2}$ film thickness increases $E_{g}$ decreases towards the band gap of bulk $\mathrm{MoS}_{2}$ $(\sim 1.3 \mathrm{eV})$. These first ellipsometric measurements of layered $\mathrm{MoS}_{2}$ films are comparable with the previous reports on $E_{g}$ of single and multilayer $\mathrm{MoS}_{2}$ derived by exfoliation. Mak et $a l^{5}$ and Lee et $a l^{7}{ }^{7}$ characterized the optical band gap energy of single and few layer mechanically exfoliated $\mathrm{MoS}_{2}$ from photoluminescence (PL) spectra and electrical measurements of $\mathrm{MoS}_{2}$ phototransistors, respectively, and found that the band gap decreased in energy with increasing $\mathrm{MoS}_{2}$ thickness. Eda et al. ${ }^{24}$ also observed a similar trend in $E_{g}$ from chemically exfoliated $\mathrm{MoS}_{2}$ using PL spectra measurements. This consistency is notable, since it implies that our vapor phase derived $\mathrm{MoS}_{2}$ has comparable optical characteristics to those of exfoliated materials. Our study further establishes SE as a viable tool for the characterization of 2D materials as it can be used to investigate $E_{g}$ of $\mathrm{MoS}_{2}$, or indeed other TMDs (e.g., $\mathrm{WTe}_{2}, \mathrm{MoSe}_{2}$, etc.), while also giving information on optical constants and thicknesses. Such measurements are fast, non-destructive, and relatively easy to perform.

In summary, SE measurements have been carried out on $\mathrm{MoS}_{2}$ thin films with different thicknesses synthesized by vapor phase sulfurization. In addition, they were characterized by XRR, UV-visible absorption spectroscopy, and Raman spectroscopy. An optical dispersion model of $\mathrm{MoS}_{2}$ was developed for SE data analysis. Values of optical constants $(n, k)$ and the thickness were determined by fitting the optical model function to the experimental data. The optical band gap energy $E_{g}$ of the $\mathrm{MoS}_{2}$ thin films was also investigated. Using the absorption coefficients of $\mathrm{MoS}_{2}$ calculated from the $k$ values, $E_{g}$ of each $\mathrm{MoS}_{2}$ film was extracted. The $E_{g}$ values of the $\mathrm{MoS}_{2}$ films show a clear dependence on the
$\mathrm{MoS}_{2}$ film thickness, indicating that thinner $\mathrm{MoS}_{2}$ films have a larger $E_{g}$. This study establishes $\mathrm{SE}$ as a promising technique for optical characterization of TMD films.

This work was supported by the SFI under Contract No. 08/CE/I1432, 12/RC/2278, and PI_10/IN.1/I3030. C.Y. and M.O. acknowledge the Embark Initiative via an Irish Research Council scholarship.

${ }^{1}$ K. S. Novoselov, D. Jiang, F. Schedin, T. J. Booth, V. V. Khotkevich, S. V. Morozov, and A. K. Geim, Proc. Natl. Acad. Sci. U.S.A. 102, 10451 (2005).

${ }^{2}$ A. H. C. Neto and K. Novoselov, Mater. Express 1, 10 (2011).

${ }^{3}$ K. S. Novoselov, Rev. Mod. Phys. 83, 837 (2011).

${ }^{4}$ Q. H. Wang, K. Kalantar-Zadeh, A. Kis, J. N. Coleman, and M. S. Strano, Nat. Nanotechnol. 7, 699 (2012).

${ }^{5}$ K. F. Mak, C. Lee, J. Hone, J. Shan, and T. F. Heinz, Phys. Rev. Lett. 105, 136805 (2010).

${ }^{6}$ B. Radisavljevic, A. Radenovic, J. Brivio, V. Giacometti, and A. Kis, Nat. Nanotechnol. 6, 147 (2011).

${ }^{7}$ H. S. Lee, S.-W. Min, Y.-G. Chang, M. K. Park, T. Nam, H. Kim, J. H. Kim, S. Ryu, and S. Im, Nano Lett. 12, 3695 (2012).

${ }^{8}$ B. Radisavljevic and A. Kis, Nature Mater. 12, 815 (2013).

${ }^{9}$ O. Lopez-Sanchez, D. Lembke, M. Kayci, A. Radenovic, and A. Kis, Nat. Nanotechnol. 8, 497 (2013).

${ }^{10}$ Y.-H. Lee, X.-Q. Zhang, W. Zhang, M.-T. Chang, C.-T. Lin, K.-D. Chang, Y.-C. Yu, J. T.-W. Wang, C.-S. Chang, L.-J. Li, and T.-W. Lin, Adv. Mater. 24, 2320 (2012)

${ }^{11}$ Y. Zhan, Z. Liu, S. Najmaei, P. M. Ajayan, and J. Lou, Small 8, 966 (2012).

${ }^{12}$ S. Najmaei, Z. Liu, W. Zhou, X. Zou, G. Shi, S. Lei, B. I. Yakobson, J.-C. Idrobo, P. M. Ajayan, and J. Lou, Nature Mater. 12, 754 (2013).

${ }^{13}$ M. Shanmugam, C. A. Durcan, and B. Yu, Nanoscale 4, 7399 (2012).

${ }^{14}$ K. Lee, R. Gatensby, N. McEvoy, T. Hallam, and G. S. Duesberg, Adv. Mater. 25, 6699 (2013).

${ }^{15}$ W. Zhang, J.-K. Huang, C.-H. Chen, Y.-H. Chang, Y.-J. Cheng, and L.-J. Li, Adv. Mater. 25, 3456 (2013).

${ }^{16} \mathrm{H}$. Fujiwara, Spectroscopic Ellipsometry: Principles and Applications (John Wiley \& Sons Ltd, Chichester, 2007), p. 81.

${ }^{17}$ V. G. Kravets, A. N. Grigorenko, R. R. Nair, P. Blake, S. Anissimova, K. S. Novoselov, and A. K. Geim, Phys. Rev. B 81, 155413 (2010).

${ }^{18}$ F. J. Nelson, V. K. Kamineni, T. Zhang, E. S. Comfort, J. U. Lee, and A. C. Diebold, Appl. Phys. Lett. 97, 253110 (2010).

${ }^{19}$ J. W. Weber, V. E. Calado, and M. C. M. van de Sanden, Appl. Phys. Lett. 97, 091904 (2010).

${ }^{20}$ A. Matkovic, U. Ralevic, M. Chhikara, M. M. Jakovljevic, D. Jovanovic, G. Bratina, and R. Gajic, J. Appl. Phys. 114, 093505 (2013).

${ }^{21}$ CompleteEASE ${ }^{T M}$ Data Analysis Manual Version 4.63, J. A. Woollam Co., Inc., Lincoln, NE, 2011, p. 45.

${ }^{22}$ C. M. Herzinger, B. Johs, W. A. McGahan, J. A. Woollam, and W. Paulson, J. Appl. Phys. 83, 3323 (1998).

${ }^{23}$ G. E. Jellison and F. A. Modine, Appl. Phys. Lett. 69, 371 (1996).

${ }^{24}$ G. Eda, H. Yamaguchi, D. Voiry, T. Fujita, M. Chen, and M. Chhowalla, Nano Lett. 11, 5111 (2011).

${ }^{25}$ H. Li, Q. Zhang, C. C. R. Yap, B. K. Tay, T. H. T. Edwin, A. Olivier, and D. Baillargeat, Adv. Funct. Mater. 22, 1385 (2012).

${ }^{26}$ F. P. Koffyberg, K. Dwight, and A. Wold, Solid State Commun. 30, 433 (1979).

${ }^{27}$ E. R. Shaaban, M. S. Abd El-Sadek, M. El-Hagary, and I. S. Yahia, Phys. Scr. 86, 015702 (2012).

${ }^{28}$ See supplementary material at http://dx.doi.org/10.1063/1.4868108 for additional SE spectral data, fitting parameter values of the T-L oscillation model, XRR details, and plots on the optical band gap extraction. 